\title{
Deformed Swept Volume Analysis to NC Machining Simulation with Cutter Deflection
}

\author{
MingC.Leu(leu@njit.edu) \\ New Jersey Institute of Technology, Newark, New Jersey, U.S.A \\ Denis Blackmore and Bilal Maiteh \\ New Jersey Institute of Technology, Newark, New Jersey, U.S.A
}

Key words: Numerical Control (NC), Machining, Simulation.

Abstract: This paper presents a deformed swept volume analysis integrated with machining mechanics for NC machining simulation with consideration of cutter deflection. This analysis uses an extended Sweep Differential Equation approach to represent the swept volumes of flexible moving objects. A mechanics model for cutter-workpiece interaction is used to compute the cutter deflection in end milling. A software program has been developed in $\mathrm{C}++$ to generate the cutter's deformed swept volume, which is subtracted from the workpiece to simulate the machining process and machined part. The simulation is shown useful to visualizing the machining process and checking machining surface errors.

\section{INTRODUCTION}

Representation of swept volume has important applications in the planning of machining process. Substantial efforts have been devoted to developing fast and accurate methods for representation of swept volumes; for example, Wang \& Wang made an early attempt to model and compute the swept volumes by applying the envelope equation formulation ${ }^{1,2}$. Blackmore and Leu introduced the Sweep Differential Equation (SDE) method for the study of swept volumes $^{3,4,5}$. This method exploits the Lie group structure of the set of Euclidean motions, thereby enabling the swept volume modeling to be reformulated as the problem of solving differential equations in the global sense. For efficient computation, they developed the sweep-envelope differential equation algorithm to generate the set of points constituting the swept-volume boundary. The SDE method was applied to NC machining simulation without cutter deflection ${ }^{6}$. Blackmore and Leu also extended the SDE method to modeling of deformed swept volumes and described how to implement it ${ }^{7,8}$.

This paper summarises the deformed swept volume analysis and its integration with machining mechanics for NC machining simulation with cutter deflection. By evaluating the linear and non-linear cutter deflections and including them in the swept volume representation, we create a more accurate $\mathrm{NC}$ verification program useful for simulating the material removal process and checking the machined part. A program is developed in $\mathrm{C}++$ for the generation of deformed swept volume based on the SDE approach. An example is given to illustrate the application of this program for milling simulation with cutter deflection.

\section{RIGID AND DEFORMED SWEPT VOLUMES}

The object $M$ under consideration is assumed to be closed with a boundary surface $\partial M$ which is piecewise smooth. In practical terms, the object considered has a smooth boundary except for a finite number of edges and vertices.

A sweep is a family of rigid motions. Mathematically, a smooth sweep $\sigma$ in which $R$ is the field of real numbers is a smooth mapping:

$$
\sigma:[0,1] \rightarrow E(n)
$$

The original version of this chapter was revised: The copyright line was incorrect. This has been corrected. The Erratum to this chapter is available at DOI: 10.1007/978-0-387-35392-0_40 
where $E(n)$ is a Lie group of Euclidean motion such that $\sigma$ at $t=0$, denoted by $\sigma_{0}$, is the identity mapping. From this definition, if we let $\sigma_{\mathrm{t}}$ represent a sweep in time $t$, it can be written as

$$
\sigma_{t}\left(\mathrm{x}_{0}\right)=\mathrm{x}(t)=\xi(t)+B(t) \mathrm{x}_{0}
$$

where: $\xi:[0,1] \rightarrow R^{n}$ and $B:[0,1] \rightarrow S O(n)$ are smooth functions representing the translation and rotation of the sweep, respectively; $\mathrm{x}_{0}$ represents any point on the boundary of object $M$ in $R^{3}$.

The set

$$
\sigma_{t}(M)=M(t)=\left\{\sigma_{t}(\mathrm{x}) ; \mathrm{x} \in M\right\}
$$

is called the $t$ section of $M$ under sweep $\sigma$. The swept volume of $M$ generated by $\sigma$ is

$$
S_{\sigma}(M)=\left\{\sigma_{t}(M): 0 \leq t \leq 1\right\}
$$

Solving equation (2) for $\mathrm{x}_{0}$ and substituting it into the time derivative of equation (2), we can obtain the sweep vector field (SVF)

$$
X_{\sigma}=\dot{\mathrm{x}}(t)=\dot{\xi}(t)+\dot{B}(t) B^{T}(t)(\mathrm{x}-\xi(t))
$$

We apply the boundary flow method (BFM) for the representation and calculation of swept volumes by partitioning the boundary of $\partial M$ at each $t$ into ingress, egress and grazing points. The set of ingress (egress) points of $M(t)$, denoted by $\partial_{-} M(t)\left(\partial_{+} M(t)\right)$, consists of all points $\mathrm{x} \in \partial M(t)$ at which $X_{\sigma}(\mathrm{x}, t)$ points into (out of) the interior of $M$. Those points that are neither ingress nor egress points are called grazing points denoted by $\partial_{0} M(t)$. Figure 1 illustrates ingress, egress, and grazing points.

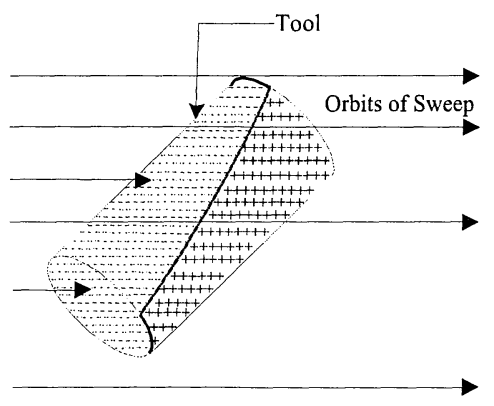

$$
\text { Ingress Point }
$$

Egress Point

$\longrightarrow$ Grazing Point

Figure 1. Object Boundary Partition.

Mathematically, we can define them in the context of tangency function. The tangency function for a sweep of object $M$ is defined as

$$
T(\mathrm{x}, t)=<X_{\sigma}(\mathrm{x}, t), N(\mathrm{x}, t)>
$$

where $\langle a, b\rangle$ denotes the inner product of $a, b$ in $R^{n}, N(\mathrm{x}, t)$ is the unit outward normal vector on the smooth part of $M$ at the point of concern. For the points on a smooth surface, they are defined as: 


$$
\begin{aligned}
& \partial_{-} M(t)=\cup\{T(\mathrm{x}, t)<0, \mathrm{x} \in M, t \in[0,1]\} \\
& \partial_{+} M(t)=\cup\{T(\mathrm{x}, t)>0, \mathrm{x} \in M, t \in[0,1]\} \\
& \partial_{0} M(t)=\cup\{T(\mathrm{x}, t)=0, \mathrm{x} \in M, t \in[0,1]\}
\end{aligned}
$$

Let the object $M$ and sweep $\sigma$ be defined as above, the boundary of the swept volume is given by $G(M) \mid W(M)$, where $G(M)=\partial_{-} M(0) \cup \partial_{+} M(1) \cup\left\{\partial_{0} M(t): 0<t<1\right\}$ is the candidate boundary set which consists of the ingress points of object $M$ at $t=0$, egress points of $M$ at $t=1$, and all the grazing points between $t=0$ and $t=1 . W(M)$ denotes the trimming set consisting of points each of which belongs to the interior of some $t$ section of $M$, and thus it does not belong to the portion of the swept-volume boundary. For the points not on a smooth surface, ingress, egress and grazing points can be also be defined by using the tangency function.

The sweep differential equation and boundary flow method can be extended to include objects experiencing deflections or deformations. Although there are several special cases of deformation which can be more easily implemented in SDE, we examine the case of general deformation. Given a piecewise smooth object under general motion and deformation, a smooth sweep with general deformation can be written as

$$
\sigma_{t}\left(\mathrm{x}_{0}\right)=\mathrm{x}(t)=\xi(t)+B(t)\left(\mathrm{x}_{0}+D\left(\mathrm{x}_{0}, t\right)\right)
$$

where $D\left(\mathrm{x}_{0}, t\right)$ denotes the deformation and $D\left(\mathrm{x}_{0}, 0\right)=\partial_{t} D\left(\mathrm{x}_{0}, 0\right)=0$ for all points on $M$.

The above equation can be rewritten in a more concise form:

$$
\sigma_{t}\left(\mathrm{x}_{0}\right)=\mathrm{x}(t)=\xi(t)+B(t) \mathrm{x}_{0}+H\left(\mathrm{x}_{0}, t\right)
$$

where $H\left(\mathrm{x}_{0}, t\right)=B(t) D\left(\mathrm{x}_{0}, t\right)$. Differentiating equation (9) with respect to $t$, we can derive the Deformed Sweep Differential Equation (i.e. SDE with deformation)

$$
X_{\sigma}=\dot{\mathrm{x}}(t)=\dot{\xi}(t)+\dot{B}(t) \mathrm{x}_{0}+\partial_{t} H\left(\mathrm{x}_{0}, t\right)
$$

The tangency function, which is used to identify the ingress, egress and grazing points, can be generated from the sweep of the initial outward normal vector $N_{0}$ of the smooth part of the object $M$. It can be expressed as

$$
\begin{aligned}
T(\mathrm{x}, t) & =<X_{\sigma}(\mathrm{x}, t), N(\mathrm{x}, t)> \\
& =<X_{\sigma}(\mathrm{x}, t),\left[\left(B(t)+\partial_{\mathrm{x}} H\left(\mathrm{x}_{0}, t\right)\right)^{-1}\right]^{T} N_{0}>
\end{aligned}
$$

A fairly straightforward method for calculating the outward normal vector field can be used. Given a surface in three-dimensional space which can be expressed by a parametric equation:

$$
\mathrm{x}(u, v)=\left[\begin{array}{l}
x(u, v) \\
y(u, v) \\
z(u, v)
\end{array}\right]
$$

Let $\mathrm{x}(u, v)$ stand for the vector of any point on this surface, the outward normal vector of the surface at this point is

$$
N(\mathrm{x}, t)=\frac{\partial \mathrm{x}(u, v)}{\partial u} \times \frac{\partial \mathrm{x}(u, v)}{\partial v}
$$

The tangency function, therefore, can be obtained from

$$
\begin{aligned}
T(\mathrm{x}, t) & =<X_{\sigma}(\mathrm{x}, t), N(\mathrm{x}, t)> \\
& =X_{x}\left(y_{u} z_{v}-y_{v} z_{u}\right)+X_{y}\left(\mathrm{x}_{v} z_{u}-\mathrm{x}_{u} z_{v}\right)+X_{z}\left(\mathrm{x}_{u} y_{v}-\mathrm{x}_{v} y_{u}\right)
\end{aligned}
$$

where $\mathrm{x}_{u}, \mathrm{x}_{v}, y_{u}, y_{v}, z_{u}$ and $z_{v}$ stand for

$$
\mathrm{x}_{u}=\frac{\partial \mathrm{x}(u, v)}{\partial u}, \mathrm{x}_{v}=\frac{\partial \mathrm{x}(u, v)}{\partial v} \cdots
$$


and $X_{x}, X_{y}$ and $X_{z}$ represent the $\mathrm{x}, y$ and $z$ components of $X_{\sigma}(\mathrm{x}, \mathrm{t})$.

\section{MODELING OF CUTTING FORCE AND CUTTER DEFLECTION IN MILLING}

The milling process is widely used in industry for shaping mechanical components, such as face milling of the top surface of an engine block or peripheral milling of a flexible aircraft turbine impeller. Models of milling are required for analysis of machining and prediction of quality of the machined part. The process models can be used for cutting force and cutter deflection prediction, machining error checking, as well as for tool breakage and wear monitoring.

Several milling force models have been proposed. Two basic ones are

I. Average force model ${ }^{9}$.

II. Instantaneous distributed force model ${ }^{10,11,12,13}$.

The average force model computes the cutting force based on the material removal rate. This method does not give accurate force at every instant. The instantaneous distributed force model is applied here. In this model, the cutting force of the tool is the sum of infinitesimal forces along the various flutes. A basic equation of this model is

$$
t_{c}=f \sin \alpha
$$

where $t_{c}$ is the instantaneous chip thickness, $f$ is the feed ( $\mathrm{mm} /$ tooth), and $\alpha$ is the angular position of the tooth in the cut, see Figure 2. By dividing the cutter along the axial depth of cut into $N_{z}$ disks, the chip area $(\Delta A)$ is associated with each disk element, $\Delta A=\Delta z t_{c}$. The tangential and radial forces due to chip loads are, respectively,

$$
\begin{aligned}
& \Delta F_{T}=K_{T}(\Delta A)=K_{T} \Delta z t_{c} \\
& \Delta F_{R}=K_{R} \Delta F_{T}
\end{aligned}
$$

where $K_{T}$ and $K_{R}$ are the cutting coefficients which vary with feed rate and axial depth of cut.

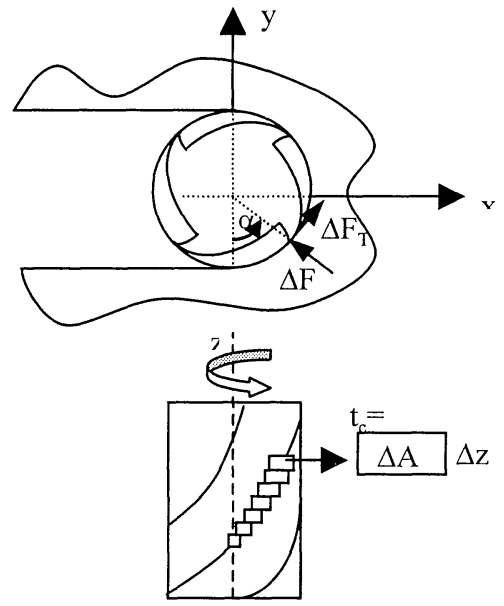

Figure 2. End Milling Force Model.

Let the cutter location be specified by the angular position of the bottom of the first flute. The chip thickness of the $i$ th axial segment of the $k$ th flute at the $j$ th angular position of the cutter is

$$
t_{c}(i, j, k)=f \sin \alpha(i, j, k)
$$


where

$$
\alpha(i, j, k)=\left[\frac{2 \pi(k-1)}{N_{f}}-\frac{2 \pi j}{N_{f} N_{\theta}}\right]+[(i-0.5) \Delta z] \frac{\tan \gamma}{R}
$$

where $\gamma$ is the helix angle, $j \in\left[1: N_{\theta}\right], N_{f}$ and $N_{\theta}$ are the numbers of flutes and angular increments, respectively. For the infinitesimal chip segments, the tangential and radial forces can be readily computed by substituting equation (16) into equation (15). The total force acting along $\mathrm{x}$ and $\mathrm{y}$ directions at the $j$ th angular position of the cutter is

$$
\begin{aligned}
& F_{x}(j)=\sum_{i=1}^{N_{3}} \sum_{k=1}^{N_{f}}-\Delta F_{R}(i, j, k) \sin \alpha(i, j, k)+\Delta F_{T}(i, j, k) \cos \alpha(i, j, k) \\
& F_{y}(j)=\sum_{i=1}^{N_{3}} \sum_{k=1}^{N_{f}} \Delta F_{R}(i, j, k) \cos \alpha(i, j, k)+\Delta F_{T}(i, j, k) \sin \alpha(i, j, k)
\end{aligned}
$$

There are two types of tool deflections in milling. The first type is linear deflection due to the compliance of the chucking device and other machine components. The second is nonlinear deflection due to the compliance of the tool itself (similar to a cantilever beam). A complete model for tool deflection must take into consideration both types of deflection.

As illustrated in Figure 3a, the linear deflection at point with distance $\mathrm{z}$ from the tool tip can be calculated as:

$$
\begin{aligned}
& \varepsilon_{x}(i, j)=E_{h} * F_{x}(j)-E_{r} * M_{y}(j) *[L-(i-0.5) \Delta z] \\
& \varepsilon_{y}(i, j)=E_{h} * F_{y}(j)+E_{r} * M_{x}(j) *[L-(i-0.5) \Delta z]
\end{aligned}
$$

Where $F_{x}(\mathrm{j})$ and $F_{y}(j)$ are the cutting forces in the $\mathrm{x}$ and $\mathrm{y}$ directions given by equation (18); $E_{h}$ and $E_{r}$ are translational and rotational compliance constants, respectively, which can be obtained experimentally; $M_{x}(\mathrm{j})$ and $M_{y}(j)$ are the moments, related to the cutting forces as

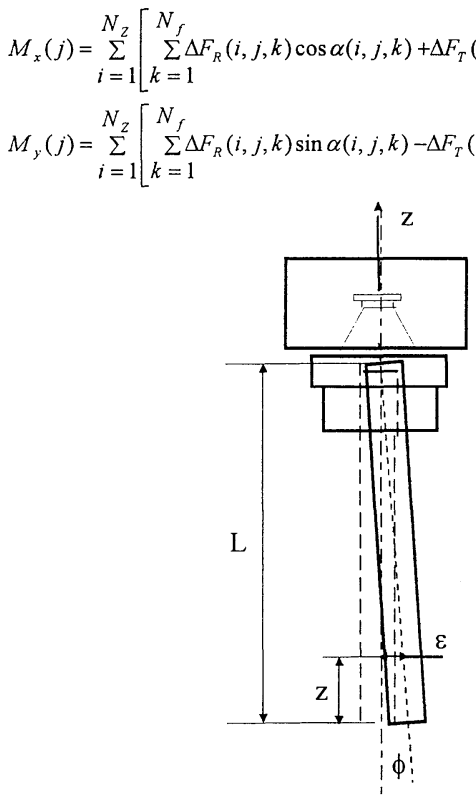

(a) Linear deflection

Figure 3. Tool Deflection Model 
For the prediction of nonlinear deflection, the end mill is simply modeled as a cantilever beam, as shown in Figure 3b. The non-linear deflection of the tool along the z-axis can be obtained as:

$$
\begin{aligned}
& \delta_{x}(i, j)=\left\{\sum_{l=1}^{i=1}\left[\sum_{k=1}^{N_{f}}-\Delta F_{R}(i, j, k) \sin \alpha(i, j, k)+\Delta F_{T}(i, j, k) \cos \alpha(i, j, k)\right] \cdot\left[(L-(i-0.5) \Delta z)^{2}(2 L+(i-l) \Delta z)\right]+\right. \\
& \left.\sum_{l=i}^{N_{z}}\left[\sum_{k=1}^{N_{f}}-\Delta F_{R}(i, j, k) \sin \alpha(i, j, k)+\Delta F_{T}(i, j, k) \cos \alpha(i, j, k)\right] \cdot\left[(L-(l-0.5) \Delta z)^{2}(2 L+(l-3 i+1) \Delta z)\right]\right\} / 6 E I \\
& \delta_{y}(i, j)=\left\{\sum_{l=1}^{i-1}\left[\sum_{k=1}^{N_{f}} \Delta F_{R}(i, j, k) \cos \alpha(i, j, k)+\Delta F_{T}(i, j, k) \sin \alpha(i, j, k)\right] \cdot\left[(L-(i-0.5) \Delta z)^{2}(2 L+(i-l) \Delta z)\right]+\right. \\
& \left.\sum_{l=i}^{N_{z}}\left[\sum_{k=1}^{N_{f}} \Delta F_{R}(i, j, k) \cos \alpha(i, j, k)+\Delta F_{T}(i, j, k) \sin \alpha(i, j, k)\right] \cdot\left[(L-(l-0.5) \Delta z)^{2}(2 L+(l-3 i+1) \Delta z)\right]\right\} / 6 E I
\end{aligned}
$$

After the linear deflection and nonlinear deflection are calculated, the total tool deflection at any point along the z-axis can be obtained as:

$$
\begin{aligned}
& D_{x}(i, j)=\varepsilon_{x}(i, j)+\delta_{x}(i, j) \\
& D_{y}(i, j)=\varepsilon_{y}(i, j)+\delta_{y}(i, j)
\end{aligned}
$$

With the cutter deflection taken into consideration, the actual machined surface is different from the ideal machined surface (without considering cutter deflection) as illustrated in Figure 4. Since a milling process consists of multiple passes, the calculation of cutting force for a pass needs to consider the machined surface error of the previous pass. The difference in the calculated cutting force due to machining error can be significant, especially if the previous pass is a rough cut and the current pass is a finish cut.

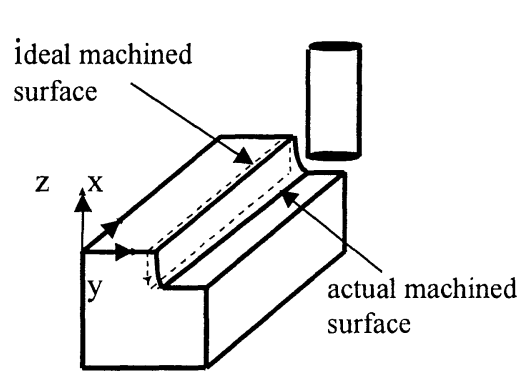

(a) Machined surface after one pass

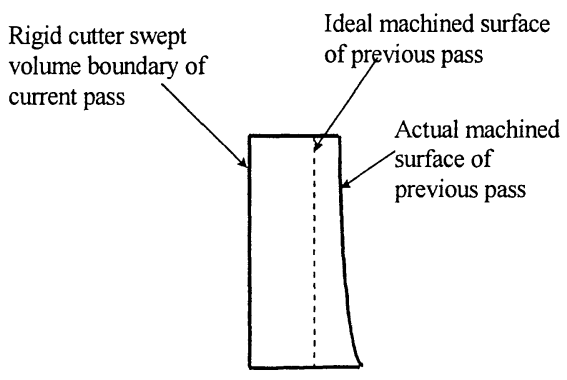

(b) Chip cross section geometry

Figure 4. Cutting Force Prediction for Multipass Cut

\section{IMPLEMENTATION IN MACHINING SIMULATION}

A computer program in $\mathrm{C}++$ for the generation of deformed cutter swept volume for end milling has been developed. The $\mathrm{CL}$ data in the simulation are obtained from a commercial $\mathrm{CAD} / \mathrm{CAM}$ system (e.g. Pro/Engineer). These data are used to generate the tool motion equations by linearly interpolating the movements of individual cutter axes. The CL data representing tool tip position and tool orientation are expressed by $\left(x_{c}, y_{c}, z_{c}, i_{c}, j_{c}, k_{c}\right)$, where $\left(x_{c}, y_{c}, z_{c}\right)$ stands for the cutter tip position and $\left(i_{c}, j_{c}, k_{c}\right)$ stands for the cutter orientation. The position of a point on the cutter during machining can be express as: 


$$
\left(\begin{array}{l}
x(t) \\
y(t) \\
z(t)
\end{array}\right)=\left(\begin{array}{l}
x_{c}(t) \\
y_{c}(t) \\
z_{c}(t)
\end{array}\right)+B \cdot\left(\begin{array}{l}
x_{0} \\
y_{0} \\
z_{0}
\end{array}\right)
$$

where $\left(x_{0}, y_{0}, z_{0}\right)$ is a vector representing the initial tool tip position, and $B$ is the rotational transform matrix which relates to the roll $(\eta)$ and pitch $(\zeta)$ angles as follows

$$
B=\left(\begin{array}{ccc}
\cos \eta & -\sin \zeta \cdot \sin \eta & \cos \zeta \cdot \sin \eta \\
0 & \cos \zeta & -\sin \zeta \\
-\sin \eta & \sin \zeta \cdot \cos \eta & \cos \zeta \cdot \cos \eta
\end{array}\right)
$$

where

$$
\begin{aligned}
& \zeta=-\tan ^{-1}\left(j_{c}(t) / \sqrt{i_{c}^{2}(t)+k_{c}^{2}(t)}\right) \\
& \eta=\tan ^{-1}\left(i_{c}(t) / k_{c}(t)\right)
\end{aligned}
$$

Thus, given one block of CL data:

$$
\left(x_{c}(0), y_{c}(0), z_{c}(0), i_{c}(0), j_{c}(0), k_{c}(0)\right) \rightarrow\left(x_{c}(1), y_{c}(1), z_{c}(1), i_{c}(1), j_{c}(1), k_{c}(1)\right),
$$

the CL data $\left(x_{c}(t), y_{c}(t), z_{c}(t), i_{c}(t), j_{c}(t), k_{c}(t)\right)$ between the initial and final locations can be calculated using linear interpolation. Substituting the obtained $\left(i_{c}(t), j_{c}(t), k_{c}(t)\right)$ into equation (26) and then (25), we obtain the rotation transform matrix. Together with the translation vector $\left(x_{c}(t), y_{c}(t), z_{c}(t)\right)$, the sweep differential equation is completely defined for the block of cutter motion.

The $\mathrm{C}++$ program for the generation of deformed cutter swept volume has been integrated with the Pro/Engineer as follows. Pro/Engineer is used for the part design. The generated CL data from the Pro/Manufacturing are read as the input to the $\mathrm{C}++$ program. The boundary of the deformed cutter swept volume in the previous pass is used to calculate the cutting force and cutter deflection for the current pass. The results are used to generate the deformed cutter swept volume for current pass. The boundary of the deformed swept volume is input to Pro/Engineer for visualization. The cutter swept volume is subtracted from the workpiece for material removal simulation.

\section{ILLUSTRATIVE EXAMPLE}

An example is given in this section to illustrate the described SDE approach for the deformed swept volume representation and its application to NC simulation. This example involves the milling of a groove on a tube clamp part. The clamp part with the groove generated on the Pro/E is shown in Figure 5a. Since the front face of the clamp is a freeform surface and a constant distance is required between the groove and this surface, the groove needs to be machined by a five-axis machine. The top protrusion of the clamp part demands the use of a long tool to machine the groove. The $\mathrm{CL}$ data are generated using Pro/Manufacturing. Figure $5 \mathrm{~b}$ shows the workpiece before machining. Figures $5 \mathrm{c}$ and $5 \mathrm{~d}$ show the workpiece during and after machining as simulated with Pro/Manufacturing. Cutter deflection is not included in these simulations because the commercial CAD/CAM package does not consider machining mechanics.

Figure $5 \mathrm{e}$ shows the undeformed cutter swept volume generated by our simulation program together with the workpiece. Figure $5 \mathrm{f}$ shows the workpiece after the material removal, obtained by substracting the undeformed cutter swept volume from the workpiece. It demonstrates that our simulation result is the same as that of the Pro/Manufacturing, i.e. Figure 5d, if we do not consider cutter deflection. 
Figure $5 \mathrm{~g}$ shows the deformed cutter swept volume together with the workpiece generated by our program. Figure $5 \mathrm{~h}$ shows the workpiece after the material removal, by subtracting the deformed cutter swept volume from the workpiece. It shows the machined part without the groove generated as desired because the tool deflection exceeds the distance between the groove and the freeform front face in the designed part. The areas of the machined surface which have errors exceeding tolerances can be indicated by colors.

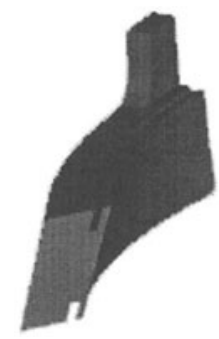

(a)

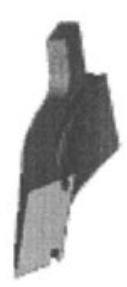

(d)

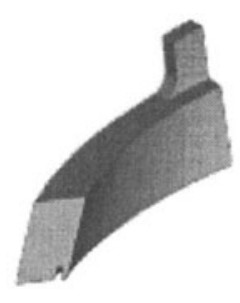

(b)

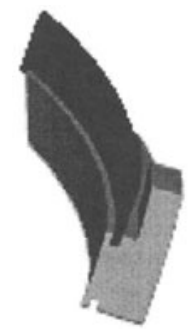

(e)

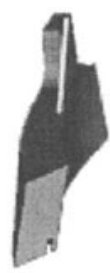

(c)

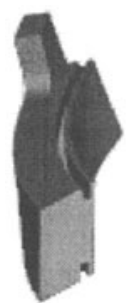

(f)

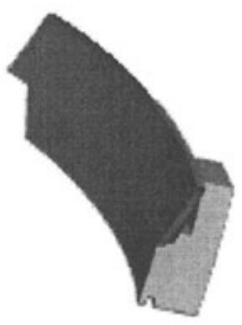

(g)

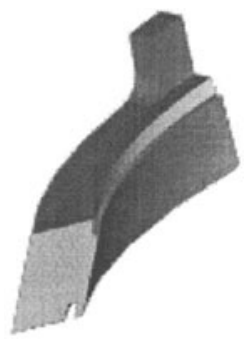

(h)

Figure 5. Simulation Example

\section{CONCLUSION}

The implementation of the Deformed Sweep Differential Equation for NC machining simulation has been described. Linear and nonlinear cutter deflections in end milling are evaluated with cutting force modeling and cutter deflection analysis. A computer program written in $\mathrm{C}++$ is developed to represent the deformed cutter swept volume. Integration of this program with the Pro/Engineer software enables the simulation of a machining process considering cutter deflection, with the CL data generated by the Pro/Manufacturing for a designed part. The applicability of the described method to simulation of industrial NC machining is demonstrated with a practical example. 


\section{ACKNOWLEDGEMENT}

This work was supported by the NSF research grant DMS-9588088. Dr. Ming C. Leu was on leave at the National Science Foundation as the Program Director for Manufacturing Machines and Equipment when the paper was perpared.

\section{REFERENCES}

1 Wang, W., Solid modeling for optimizing metal removal of three dimensional NC end milling, Journal of Manufacturing Systems,1988, 7, 57-65.

2 Wang, W., and Wang, K.K., Geometric modeling for swept volumes of moving solids, IEEE Computer Graphics \& Application, 1986, 6, 8-17.

3 Blackmore, D., Leu, M.C., and Wang, K.K., Applications of flows and envelopes to NC machining, CIRP Annals, 1992, 42, 493-496.

4 Blackmore, D., Leu, M.C., and Wang, L., The sweep-envelope differential equation algorithm and its application to NC machining verification, Computer-Aided Design, 1996, 29, 629-637

5 Blackmore, D., and Leu, M.C., Analysis of swept volume via Lie groups and differential equations, International Journal of Robotics Research, 1992, 11, 516-537.

6 Leu, M.C., Wang, L., and Blackmore, D., A verification program for 5-axis NC machining with general APT tools, Annals of CIRP, 1997, 46, 419-424.

7 Blackmore, D., Leu, M.C., and Shih, F., Analysis and modeling of deformed swept volumes, Computer-Aided Design, 1994, 26, 315-325.

8 Leu, M.C., Lu, F., and Blackmore, D., Simulation of NC machining with cutter deflection by modeling deformed swept volumes, Annals of CIRP, 1998, 47,441-446.

9 Smith, S. and Tlustry, J., An overview of modeling and simulation of the milling process, ASME Journal of Engineering for Industry, 1991, 113, 169-175.

10 Martellotti, M., An analysis of the end milling process, Transactions of ASME, 1941, 63, 677-700.

11 Martellotti, M., An analysis of the end milling process, part II-down milling, Transactions of ASME, 1945, 67, 233-251.

12 Kline, W.A., and DeVor, R.E., The prediction of surface accuracy in end milling, ASME Journal of Engineering for Industry, 1982, 104, 272-278.

13 Takata, S., Tsai, M.D., Sata, T., and Inui, M., A cutting simulation system for machinability evaluation using a workpiece model, Annals of CIRP, 1989, 38, 417-420. 